\title{
3 Towards the Development of a Pan-European Stochastic Precipitation Dataset
}

5 L.-A. Kautz ${ }^{1}$, F. Ehmele ${ }^{1}$, P. Ludwig ${ }^{1}$, H. S. Lentink ${ }^{1}$, F. D. Kelemen ${ }^{1,2}$, M. Kadlec ${ }^{3}$, and J. G. Pinto ${ }^{1}$

\author{
${ }^{1}$ Institute of Meteorology and Climate Research, Karlsruhe Institute of Technology, Karlsruhe, Germany \\ ${ }^{2}$ now at: Institute for Atmospheric and Environmental Sciences, Goethe University Frankfurt, Frankfurt am Main, \\ Germany \\ ${ }^{3}$ Impact Forecasting, Aon, Prague, Czech Republic
}

0. Contents of this file

1. Evaluation of bias correction methods in a climatological perspective

2. Added value of bias correction for extreme precipitation events

Tables S1 to S5

Figures S1 to S3

\section{1. Evaluation of bias correction methods in a climatological perspective}

16 Tables S1, S2, S3, and S4 contain the results of the evaluation of the tested bias correction methods

17 for the Elbe, Oder, Rhine, and Vistula catchments. The results are already discussed in Sect. 4 of the

18 main paper.

\section{2. Added value of bias correction for extreme precipitation events}

20 Figure S1 shows the time evolution of the Rhine flood in May 1983. Figures S2 and S3 show the

21 precipitation pattern for the 1997 and 2002 flooding events. The results are already discussed in

22 Sect. 5 of the main paper. Table S5 shows the added value of bias correction for the top ten river

23 floods between 1979 and 2010 for ERA20C-CCLM. 
24 Table S1. Root mean square error (RMSE), Pearson's correlation coefficients (R) and Taylor's skill 25 scores (S) for the raw model runs plus for the model runs with different bias corrections just for the 26 Elbe catchment: linear scaling (LS), local intensity scaling (LOCI), power transformation (PT), 27 empirical quantile mapping (EQM), and quantile mapping with gamma distribution (GQM). Results 28 of ERAI-CCLM based on RPI and of ERA20C-CCLM on RPII. Best values of each measure are 29 print in bold.

\begin{tabular}{l|ccc|ccc}
\hline & \multicolumn{3}{|c|}{ ERAI-CCLM } & \multicolumn{3}{c}{ ERA20C-CCLM } \\
& RMSE & $\mathrm{R}$ & $\mathrm{S}$ & RMSE & $\mathrm{R}$ & $\mathrm{S}$ \\
\hline raw & 2.178 & 0.641 & 0.950 & 2.454 & 0.509 & 0.952 \\
LS $_{\text {monthly }}$ & 2.224 & 0.640 & 0.948 & 2.551 & 0.506 & 0.947 \\
LS $_{\text {seasonal }}$ & 2.220 & 0.641 & 0.948 & 2.549 & 0.505 & 0.946 \\
LS $_{\text {half-yearly }}$ & 2.225 & 0.637 & 0.946 & 2.542 & 0.503 & 0.946 \\
LOCI $_{\text {monthly }}$ & 2.268 & 0.640 & 0.944 & 2.605 & 0.505 & 0.941 \\
LOCI $_{\text {seasonal }}$ & 2.265 & 0.640 & 0.945 & 2.603 & 0.504 & 0.941 \\
LOCI $_{\text {half-yearly }}$ & 2.269 & 0.637 & 0.943 & 2.596 & 0.502 & 0.941 \\
PT $_{\text {monthly }}$ & 2.105 & 0.659 & 0.960 & 2.406 & 0.528 & 0.965 \\
PT $_{\text {seasonal }}$ & 2.108 & 0.657 & 0.959 & 2.409 & 0.527 & 0.964 \\
PT $_{\text {half-yearly }}$ & 2.110 & 0.656 & 0.958 & 2.409 & 0.526 & 0.963 \\
EQM $_{\text {monthly }}$ & 2.154 & $\mathbf{0 . 6 6 2}$ & $\mathbf{0 . 9 6 1}$ & 2.466 & $\mathbf{0 . 5 3 1}$ & $\mathbf{0 . 9 6 5}$ \\
EQM $_{\text {seasonal }}$ & 2.164 & 0.659 & 0.959 & 2.476 & 0.528 & 0.962 \\
EQM $_{\text {half-yearly }}$ & 2.177 & 0.655 & 0.957 & 2.482 & 0.525 & 0.961 \\
GQM $_{\text {monthly }}$ & $\mathbf{2 . 1 0 1}$ & 0.657 & 0.958 & $\mathbf{2 . 4 0 1}$ & 0.527 & 0.964 \\
GQM $_{\text {seasonal }}$ & 2.108 & 0.655 & 0.957 & 2.410 & 0.524 & 0.962 \\
GQM $_{\text {half-yearly }}$ & 2.116 & 0.652 & 0.955 & 2.413 & 0.521 & 0.959 \\
\hline
\end{tabular}

30 Table S2. As in Tab. S1, but for the Oder catchment.

\begin{tabular}{l|ccc|ccc}
\hline & \multicolumn{3}{|c|}{ ERAI-CCLM } & \multicolumn{3}{c}{ ERA20C-CCLM } \\
& RMSE & $\mathrm{R}$ & $\mathrm{S}$ & RMSE & $\mathrm{R}$ & $\mathrm{S}$ \\
\hline raw & 2.546 & 0.538 & 0.947 & 2.997 & 0.320 & 0.879 \\
LS & 2.642 & 0.547 & 0.953 & 2.976 & 0.429 & 0.949 \\
LS $_{\text {seasonal }}$ & 2.650 & 0.542 & 0.950 & 2.963 & 0.425 & 0.948 \\
LS $_{\text {half-yearly }}$ & 2.655 & 0.538 & 0.948 & 2.954 & 0.424 & 0.948 \\
LOCI $_{\text {monthly }}$ & 2.445 & 0.548 & 0.932 & 2.767 & 0.429 & 0.952 \\
LOCI $_{\text {seasonal }}$ & 2.457 & 0.543 & 0.928 & 2.764 & 0.425 & 0.948 \\
LOCI $_{\text {half-yearly }}$ & 2.462 & 0.539 & 0.925 & 2.759 & 0.423 & 0.946 \\
PT $_{\text {monthly }}$ & 2.455 & $\mathbf{0 . 5 6 4}$ & $\mathbf{0 . 9 6 1}$ & 2.726 & $\mathbf{0 . 4 4 9}$ & $\mathbf{0 . 9 6 8}$ \\
PT $_{\text {seasonal }}$ & 2.462 & 0.562 & 0.959 & 2.727 & 0.448 & 0.967 \\
PT $_{\text {half-yearly }}$ & 2.468 & 0.560 & 0.958 & 2.728 & 0.447 & 0.966 \\
EQM $_{\text {monthly }}$ & $\mathbf{2 . 4 3 0}$ & 0.564 & 0.956 & $\mathbf{2 . 7 2 6}$ & 0.448 & 0.967 \\
EQM $_{\text {seasonal }}$ & 2.460 & 0.555 & 0.951 & 2.739 & 0.443 & 0.963 \\
EQM $_{\text {half-yearly }}$ & 2.469 & 0.551 & 0.949 & 2.747 & 0.440 & 0.961 \\
GQM $_{\text {monthly }}$ & 2.465 & 0.561 & 0.959 & 2.737 & 0.447 & 0.967 \\
GQM $_{\text {seasonal }}$ & 2.490 & 0.554 & 0.955 & 2.748 & 0.442 & 0.963 \\
GQM $_{\text {half-yearly }}$ & 2.498 & 0.550 & 0.953 & 2.748 & 0.439 & 0.961 \\
\hline
\end{tabular}


31 Table S3. As in Tab. S1, but for the Rhine catchment.

\begin{tabular}{l|ccc|ccc}
\hline & \multicolumn{3}{|c|}{ ERAI-CCLM } & \multicolumn{3}{c}{ ERA20C-CCLM } \\
& RMSE & $\mathrm{R}$ & $\mathrm{S}$ & RMSE & $\mathrm{R}$ & $\mathrm{S}$ \\
\hline raw & 2.693 & 0.745 & 0.945 & 2.992 & 0.647 & 0.951 \\
LS $_{\text {monthly }}$ & 2.638 & 0.743 & 0.947 & 3.032 & 0.643 & 0.949 \\
LS $_{\text {seasonal }}$ & 2.635 & 0.743 & 0.948 & 3.035 & 0.642 & 0.948 \\
LS $_{\text {half-yearly }}$ & 2.630 & 0.743 & 0.948 & 3.026 & 0.642 & 0.948 \\
LOCI $_{\text {monthly }}$ & 2.691 & 0.743 & 0.945 & 3.094 & 0.643 & 0.946 \\
LOCI $_{\text {seasonal }}$ & 2.688 & 0.743 & 0.945 & 3.096 & 0.642 & 0.946 \\
LOCI $_{\text {half-yearly }}$ & 2.683 & 0.743 & 0.945 & 3.087 & 0.641 & 0.946 \\
PT $_{\text {monthly }}$ & 2.526 & 0.761 & $\mathbf{0 . 9 5 7}$ & 2.916 & 0.663 & $\mathbf{0 . 9 6 0}$ \\
PT $_{\text {seasonal }}$ & 2.534 & 0.759 & 0.956 & 2.919 & 0.662 & 0.960 \\
PT $_{\text {half-yearly }}$ & 2.536 & 0.759 & 0.956 & 2.921 & 0.661 & 0.960 \\
EQM $_{\text {monthly }}$ & 2.616 & $\mathbf{0 . 7 6 3}$ & 0.955 & 3.008 & $\mathbf{0 . 6 6 5}$ & 0.959 \\
EQM $_{\text {seasonal }}$ & 2.627 & 0.761 & 0.954 & 3.016 & 0.663 & 0.958 \\
EQM $_{\text {half-yearly }}$ & 2.635 & 0.759 & 0.953 & 3.025 & 0.660 & 0.957 \\
GQM $_{\text {monthly }}$ & $\mathbf{2 . 5 2 3}$ & 0.759 & 0.956 & $\mathbf{2 . 9 1 0}$ & 0.661 & 0.959 \\
GQM $_{\text {seasonal }}$ & 2.536 & 0.757 & 0.955 & 2.919 & 0.659 & 0.958 \\
GQM $_{\text {half-yearly }}$ & 2.542 & 0.756 & 0.955 & 2.915 & 0.659 & 0.958 \\
\hline
\end{tabular}

32

33 Table S4. As in Tab. S1, but for the Vistula catchment.

\begin{tabular}{l|ccc|ccc}
\hline & \multicolumn{3}{|c|}{ ERAI-CCLM } & \multicolumn{3}{c}{ ERA20C-CCLM } \\
& RMSE & $\mathrm{R}$ & $\mathrm{S}$ & RMSE & $\mathrm{R}$ & $\mathrm{S}$ \\
\hline raw & 2.545 & 0.533 & 0.952 & 2.986 & 0.283 & 0.849 \\
LS $_{\text {monthly }}$ & 2.648 & 0.534 & 0.956 & 2.833 & 0.438 & 0.940 \\
LS $_{\text {seasonal }}$ & 2.632 & 0.535 & $\mathbf{0 . 9 5 6}$ & 2.816 & 0.437 & 0.942 \\
LS $_{\text {half-yearly }}$ & 2.635 & 0.533 & 0.956 & 2.806 & 0.435 & 0.942 \\
LOCI $_{\text {monthly }}$ & 2.421 & 0.531 & 0.853 & 2.590 & 0.433 & 0.939 \\
LOCI $_{\text {seasonal }}$ & 2.419 & 0.531 & 0.849 & 2.583 & 0.432 & 0.937 \\
LOCI $_{\text {half-yearly }}$ & 2.421 & 0.530 & 0.848 & 2.579 & 0.429 & 0.933 \\
PT $_{\text {monthly }}$ & 2.425 & $\mathbf{0 . 5 5 0}$ & 0.944 & 2.524 & $\mathbf{0 . 4 6 5}$ & $\mathbf{0 . 9 6 5}$ \\
PT $_{\text {seasonal }}$ & 2.428 & 0.548 & 0.943 & 2.526 & 0.463 & 0.964 \\
PT $_{\text {half-yearly }}$ & 2.426 & 0.549 & 0.943 & 2.529 & 0.461 & 0.962 \\
EQM $_{\text {monthly }}$ & $\mathbf{2 . 4 0 5}$ & 0.546 & 0.926 & $\mathbf{2 . 5 1 8}$ & 0.462 & 0.962 \\
EQM $_{\text {seasonal }}$ & 2.414 & 0.543 & 0.924 & 2.529 & 0.458 & 0.959 \\
EQM $_{\text {half-yearly }}$ & 2.409 & 0.544 & 0.924 & 2.539 & 0.453 & 0.956 \\
GQM $_{\text {monthly }}$ & 2.442 & 0.547 & 0.946 & 2.541 & 0.461 & 0.963 \\
GQM $_{\text {seasonal }}$ & 2.447 & 0.545 & 0.945 & 2.547 & 0.458 & 0.961 \\
GQM $_{\text {half-yearly }}$ & 2.442 & 0.546 & 0.944 & 2.555 & 0.453 & 0.957 \\
\hline
\end{tabular}


34 Table S5. Top 10 events of the five investigated river catchments for the time period from 1979 to 35 2010. The events are identified as the ten highest catchment-averaged 7-day running means of 36 precipitation from E-OBS. The indicated dates (YYYY-MM-DD) address the timing of the peak 37 value. Events where bias correction (EQM monthly) do not have an added value and the ERA20C38 CCLM shows large differences towards observations are printed in italic (-) Events which are 39 already captured well in ERA20C-CCLM, so that bias correction cannot lead to a further 40 improvement, are printed standard (0). Events where bias correction has an added value are in bold 41 print (+). The underlined events are very well captured.

\begin{tabular}{c|cl|cl|cc|cc|cc|cc}
\hline Top 10 & Rhine & & Danube & & Elbe & & Oder & & Vistula & \\
\hline 1 & $\mathbf{1 9 9 1 - 1 2 - 1 9}$ & $(+)$ & $2002-08-09$ & $(-)$ & $2002-08-09$ & $(-)$ & $1997-07-06$ & $(-)$ & $2010-09-03$ & $(-)$ \\
2 & $1998-10-26$ & $(0)$ & $\underline{\mathbf{1 9 7 9 - 0 6 - 1 6}}$ & $(+)$ & $1983-08-04$ & $(-)$ & $\mathbf{1 9 8 1 - 0 7 - 2 1}$ & $(+)$ & $2006-08-09$ & $(-)$ \\
3 & $1986-10-22$ & $(0)$ & $\underline{\mathbf{1 9 8 1 - 0 7 - 1 8}}$ & $(+)$ & $1986-12-30$ & $(-)$ & $\mathbf{1 9 9 6 - 0 7 - 0 9}$ & $(+)$ & $1992-09-04$ & $(-)$ \\
4 & $\underline{1995-01-25}$ & $(0)$ & $\mathbf{2 0 0 9 - 0 6 - 2 2}$ & $(+)$ & $1997-07-18$ & $(-)$ & $1995-08-31$ & $(-)$ & $2009-06-22$ & $(-)$ \\
5 & $\underline{1990-02-13}$ & $(0)$ & $\mathbf{1 9 8 5 - 0 8 - 0 4}$ & $(+)$ & $\mathbf{1 9 8 1 - 0 7 - 2 1}$ & $(+)$ & $\mathbf{2 0 0 1 - 0 7 - 1 8}$ & $(+)$ & $1997-07-06$ & $(-)$ \\
6 & $\underline{1993-12-22}$ & $(0)$ & $\underline{\mathbf{1 9 9 5 - 0 8 - 2 9}}$ & $(+)$ & $\mathbf{1 9 9 8 - 1 0 - 2 9}$ & $(+)$ & $2010-07-20$ & $(-)$ & $2007-07-07$ & $(0)$ \\
7 & $\underline{1983-05-23}$ & $(-)$ & $\underline{\mathbf{2 0 0 2 - 0 3 - 2 1}}$ & $(+)$ & $\underline{2010-09-26}$ & $(-)$ & $2002-08-13$ & $(-)$ & $1995-09-01$ & $(-)$ \\
8 & $\underline{\mathbf{2 0 0 4 - 0 1 - 1 1}}$ & $(0)$ & $\underline{2006-08-04}$ & $(-)$ & $\mathbf{1 9 9 5 - 0 8 - 2 9}$ & $(+)$ & $2006-08-05$ & $(-)$ & $2001-07-23$ & $(-)$ \\
9 & $\underline{\mathbf{1 9 8 4 - 0 9 - 0 7}}$ & $(+)$ & $\underline{\mathbf{2 0 0 3 - 1 0 - 0 6}}$ & $(+)$ & $\mathbf{1 9 9 6 - 0 7 - 0 8}$ & $(+)$ & $\underline{1986-06-02}$ & $(0)$ & $2010-05-15$ & $(-)$ \\
10 & $\underline{\mathbf{1 9 8 3 - 1 1 - 2 7}}$ & $(0)$ & $\mathbf{1 9 9 1 - 0 7 - 3 0}$ & $(+)$ & $\underline{\mathbf{2 0 0 1 - 0 9 - 1 0}}$ & $(0)$ & $1980-07-06$ & $(0)$ & $1980-10-11$ & $(-)$ \\
\hline
\end{tabular}

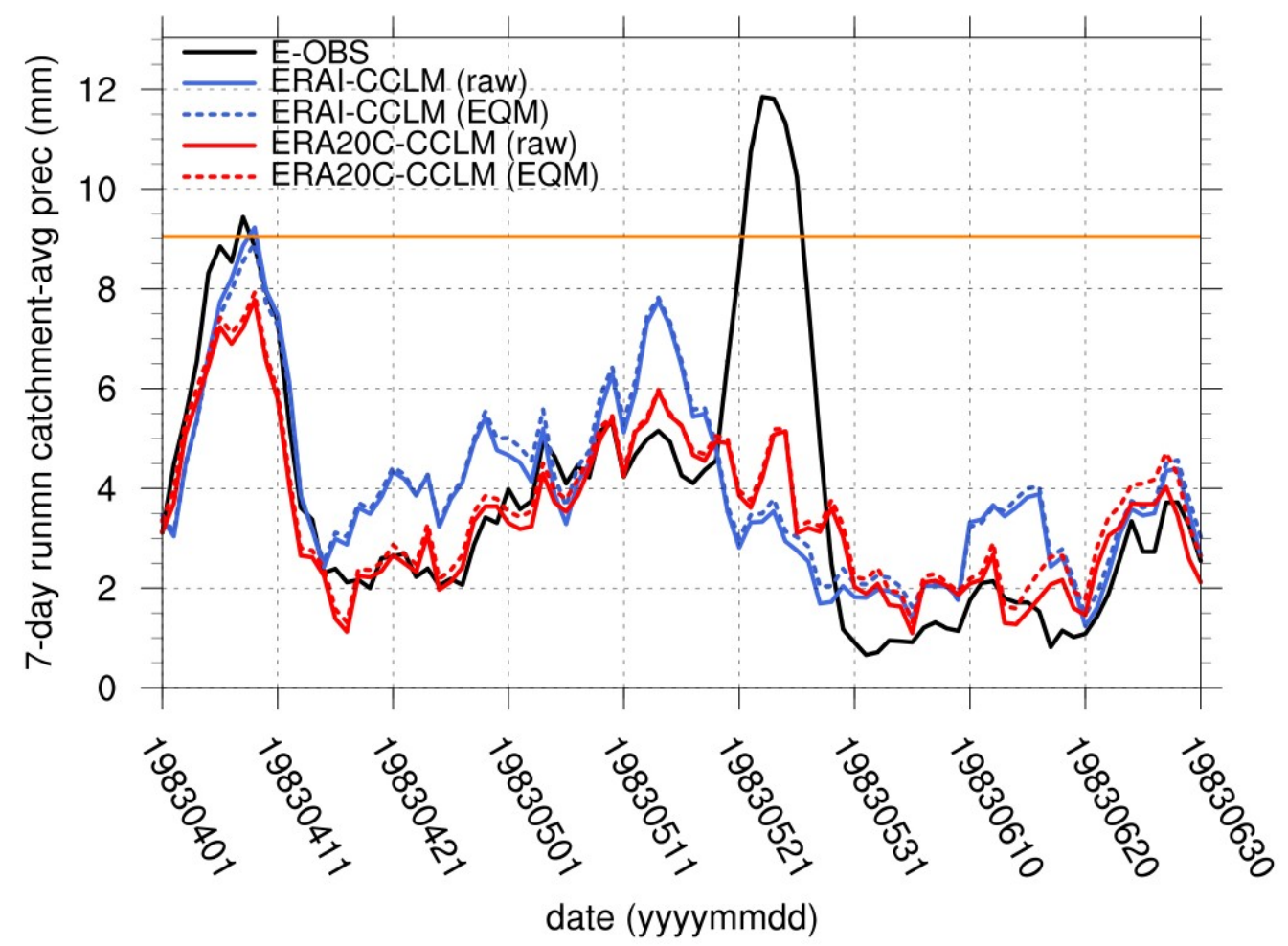

42 Figure S1. Time series of the 7-day running mean (in $\mathrm{mm}$ ) averaged over the Rhine catchment. The orange line marks the $99^{\text {th }}$ percentile of the daily precipitation sum from E-OBS based on the time period between 1979 and 2010. 
a) E-OBS

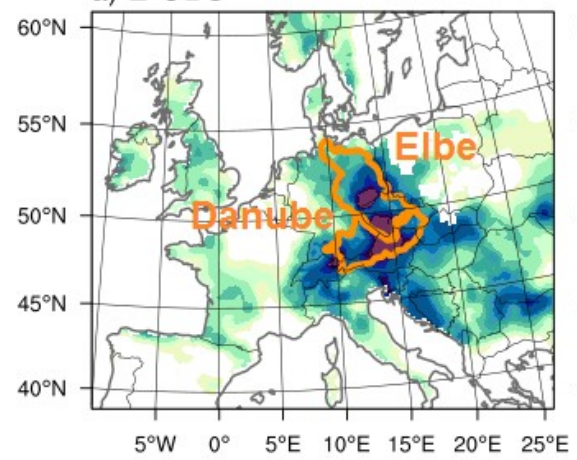

b) ERAI-CCLM (EQM)

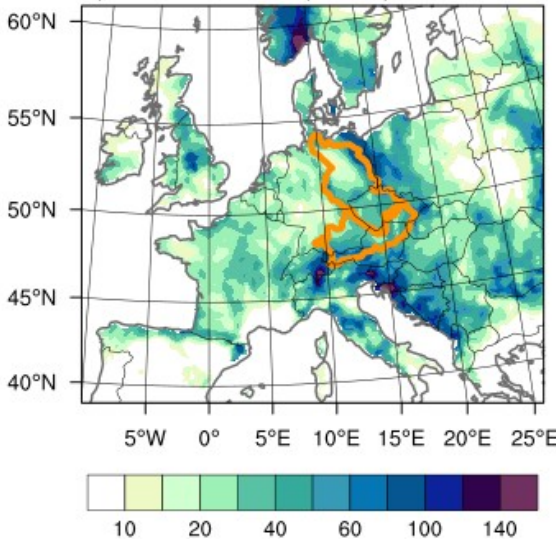

c) ERA20C-CCLM (EQM)

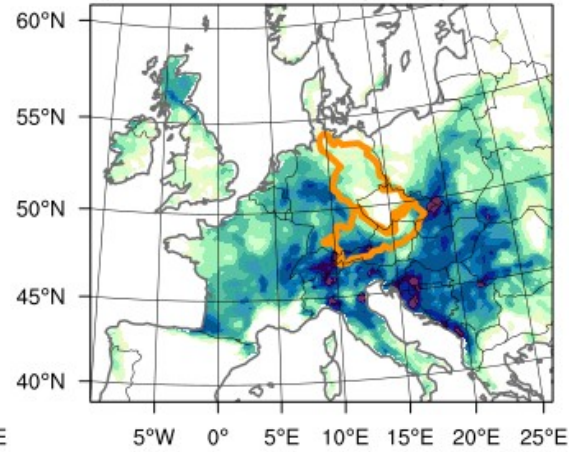

45 Figure S2. 7-day precipitation sum (in mm) for 7 to 13 August 2002 in a) E-OBS, b) ERAI-CCLM, 46 and c) ERA20C-CCLM. Orange lines mark the relevant river catchments.

a) E-OBS

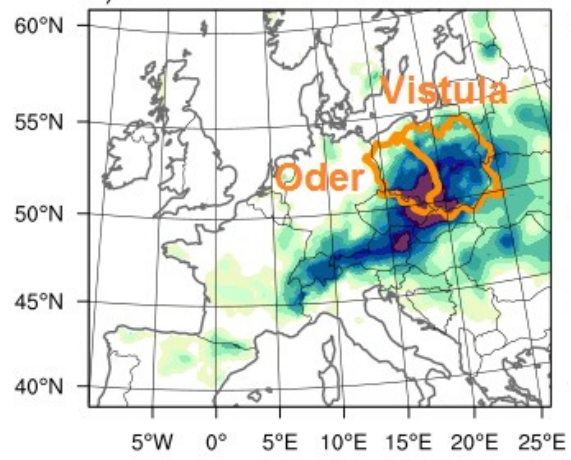

b) ERAI-CCLM (EQM)

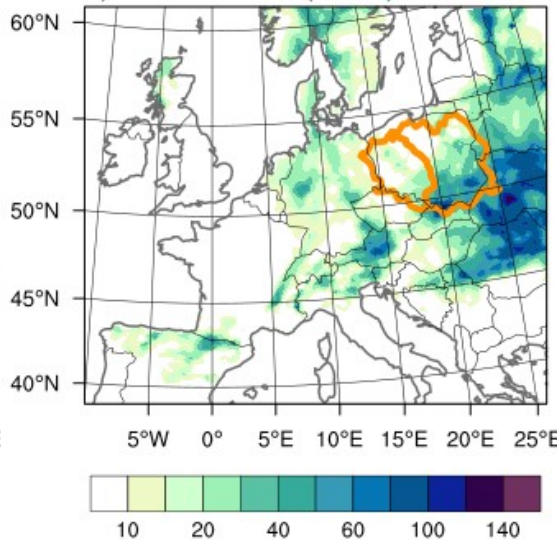

c) ERA20C-CCLM (EQM)

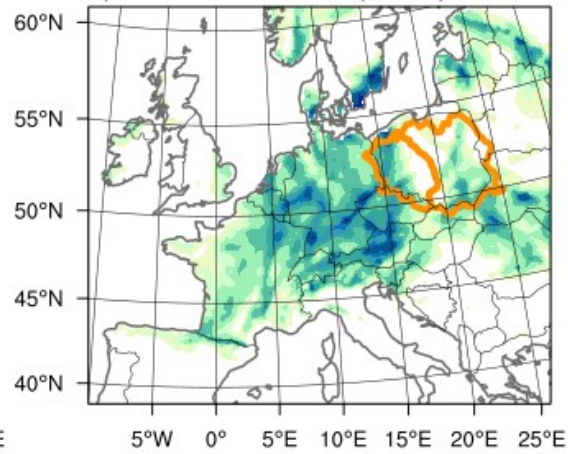

47 Figure S3. 7-day precipitation sum (in mm) for 3 to 8 July 1997 in a) E-OBS, b) ERAI-CCLM, and 48 c) ERA20C-CCLM. Orange lines mark the relevant river catchments. 